Title:

Author(s):

Submitted to:

\section{FORCE PROTECTION}

\section{RECEIVED \\ DEC 161997}

Gregory H. Canavan, P-DO

For Report of the Defense Science Board Study of

Transnational Terrorism

Date: September 1997

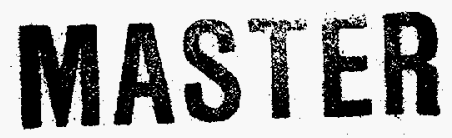

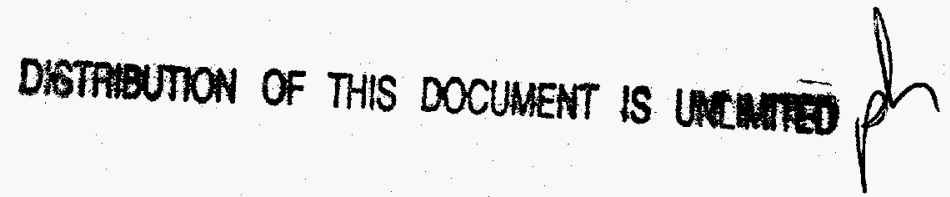

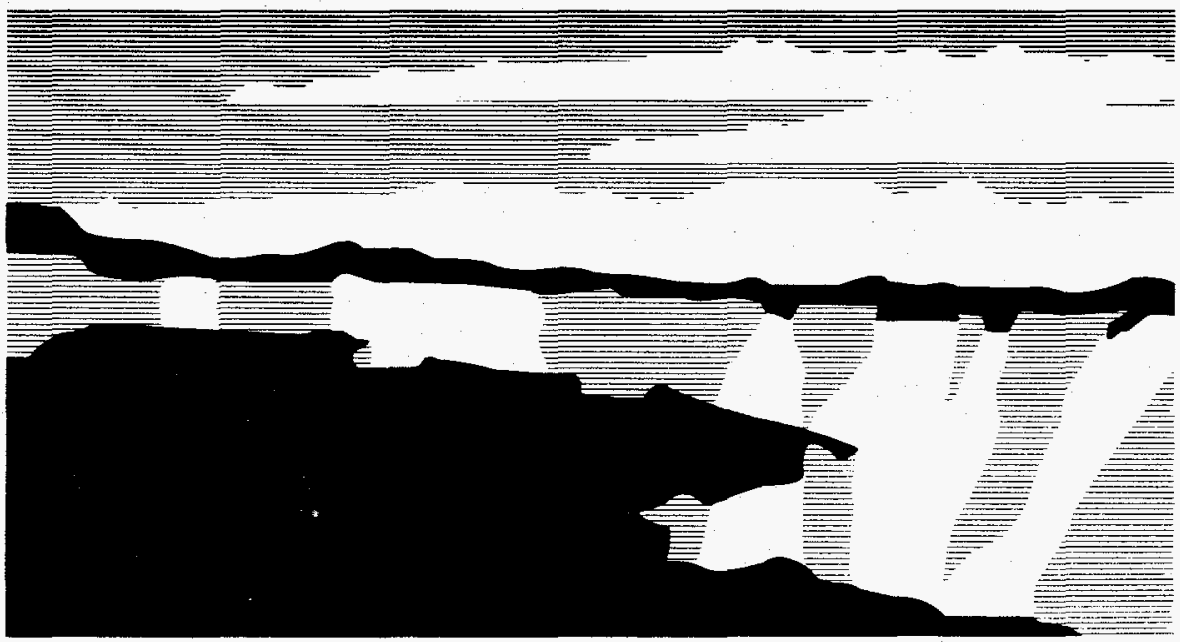

Los Alamos National Laboratory, an affirmative action/equal opportunity employer, is operated by the University of California for the U.S. Department of Energy under contract W-7405-ENG-36. By acceptance of this article, the publisher recognizes that the U.S. Government retains a nonexclusive, royalty-free license to publish or reproduce the published form of this contribution, or to allow others to do so, for U.S. Government purposes. The Los Alamos National Laboratory requests that the publisher identify this article as work performed under the auspices of the U.S. Department of Energy. 


\section{DISCLAIMER}

This report was prepared as an account of work sponsored by an agency of the United States Government. Neither the United States Government nor any agency thereof, nor any of their employees, makes any warranty, express or implied, or assumes any legal liability or responsibility for the accuracy, completeness, or usefulness of any information, apparatus, product, or process disclosed, or represents that its use would not infringe privately owned rights. Reference herein to any specific commercial product, process, or service by trade name, trademark, manufacturer, or otherwise does not necessarily constitute or imply its endorsement, recommendation, or favoring by the United States Government or any agency thereof. The views and opinions of authors expressed herein do not necessarily state or reflect those of the United States Government or any agency thereof. 


\section{DISCLAMIER}

Portions of this document may be illegible in electronic image produets. Ingges are produced from the best available original docement. 


\section{FORCE PROTECTION}

What are we trying to do?

The DoD needs the capability to do rapid, continuous inspection of vehicles entering military facilities or compounds for concealed high explosives and the capability to perform rapid inspection of interior facilities if perimeter security is breached.

How is it done now?

The military now relies primarily on physical inspection by soldiers and canine olfaction at portals and perimeters. It has no significant search rate for large areas. Dogs are the classic trace detection system. They can detect the characteristic scent of explosive and/or the other ingredients in the explosive formulation. They are used successfully to do land mine clearance and explosive detection, but have operational problems for routine screening, where they lose interest in the task, which may not be readily detectable.

There are significant differences between civil and military capabilities. The U.S. FAA has responded to concern over airline passenger safety over the last few decades by developing a number of technologies for explosive detection that are widely deployed and continually upgraded. These technologies are used below to discuss potential improvements to current DoD capabilities (L. Malotky, "Advances in Security Technology," FAA report, June 1995).

$x$-ray detection systems are derivatives of medical imaging systems. They have been used for several decades. The first $\mathrm{x}$-ray security systems employed simple $\mathrm{x}$-ray attenuation to produce a shadowgraph of the object being screened. That works well for high-contrast targets such as handguns but is not as effective for unstructured targets like sheets of explosives. Transmission Xray screening is currently being used for customs contraband screening of trucks and cargo containers, but it is still limited by the difficulty of interpreting the signatures from bulk explosives.

In the early 1990 s, two-energy $x$-ray devices were used to differentiate high atomic number materials, like the iron of weapons, from the absorption of low atomic number explosives. Dual energy systems are in use today in baggage screening systems in which an operator observes the image. As computing power increased and became more affordable, it became possible to develop automated dual energy image explosive detection systems. A significant number of these automatic systems, currently costing about $\$ 350,000$, are being used to screen checked baggage in the United States and Europe.

$x$-ray tomography evolved from medical applications requiring precise, non-destructive, two- and three-dimensional imaging of tissue. A computer tomography system, the InVision CTX5000, was submitted to the FAA for certification as an automated Explosives Detection System, underwent formal certification testing, and was certified in 1994. It takes selected tomographic slices through the object being screened and uses density and size information generated to make a decision on the presence of an explosive threat. It has demonstrated the ability to detect threat quantities of a broad range of commercial and military explosives. It is now deployed in airports in the US and abroad, and the FAA is purchasing over 50 units at about $\$ 900,000$ each and providing them to air carriers to screen checked baggage.

Thermal neutrons. In the 1980s thermal neutron analysis was explored for the detection of explosives concealed in checked baggage and cargo. Radioactive decay and electronic neutron generators were used. The thermalized neutrons react with nitrogen atoms in all commercial and military explosives to give a $10.8 \mathrm{MeV}$ gamma ray, which stands out from the background, allowing an estimation of the amount of nitrogen present. However, innocent objects in baggage with high nitrogen densities cause false alarms. Following PA 103, thermal neutron systems were deployed in six airports to collect operational information. Their performance and operational availability were good, but they were not accepted by air carriers because of system size, cost and limited ability to address explosives smaller than about $1 \mathrm{~kg}$.

Fast neutrons are scattered by atoms they encounter. The energy of the resulting gamma rays are characteristic of the element, which allows the operator to do an in situ elemental analysis. Explosives can be recognized by their characteristic elemental ratios of oxygen, carbon and nitrogen. Elements present in improvised explosives, e.g. chlorine and very high levels of oxygen, may assist in the detection of improvised explosives. 
Fast neutrons have been explored in three different geometries. A sealed tube neutron generator with an imaging alpha detector was developed in the early 1980 s, in which the collision of a tritium atom on deuterium produces a $14 \mathrm{MeV}$ neutron and a colinear alpha particle. The alpha particle can be imaged and the position of each neutron of interest as a function of time predicted. The timed arrival of a gamma ray from the interaction of the fast neutron with an atom allows one to determine its location in space.

In pulsed fast neutron analysis, neutrons are created in narrow bursts about 1 nanosecond wide, and the gamma ray detectors are collimated to look at one line. The time of arrival of a gamma ray tells the operator where the element is along the line of propagation. The energies of the gamma ray indicate which elements are in the beam. Transmission shadowgraphs can also be done using broad energy range fast neutrons. Specific elements. in the beam scatter selected neutron energies. The determinations of which energies are absent allow the determination of which elements are in the beam line and potentially whether explosives are present.

These three approaches are all in the experimental stage. The pulsed fast neutron approach is the most mature. An operational prototype is under construction. It has been used in the laboratory to screen luggage and cargo in 20 foot containers for explosives.

Quadrupole Resonance is the emerging electromagnetic approach. Rather than ionizing radiation it uses an alternating high-frequency magnetic field. An applied 3.5 MHz RF magnetic field interacts with the nitrogen molecules in explosives. Because of their crystalline structure, the field interacts with the atoms only at certain, unique frequencies. Due to their specificity, detection is good and false alarm rates are small. However, because of the specificity of the interaction frequencies, the detection system must interrogate the sample with the correct frequency and pulse train shape for each explosive of interest.

Laboratory testing was accomplished with a large scale coil (300 liters) quadrupole resonance system using 300 lost bags, 100 of which were loaded with threat and sub-threat quantities of explosives. The technology is available commercially in the form of small systems to interrogate parcels for RDX and PETN; other explosives are being added. Quadrupole resonance is the result of research in several countries and partnerships between government and industry. It requires neither massive radiation shielding nor sophisticated image analysis software. It would fit well into an integrated security screening system in which several technologies worked together.

Trace detection systems have been employed operationally protecting a variety of facilities for at least 15 years. The systems of today are capable of detecting traces of explosives present on a variety of concealments. Dogs are the classic trace detection system; they can detect the scent of explosive and/or other ingredients in the explosive formulation. They are used successfully for explosive detection but have operational problems for routine screening. Scientists have been working to develop an electronic equivalent to the dog's nose since the early 1970's. Current technology is capable of simultaneously detecting and identifying less than one nanogram of RDX or PETN, volatile explosives, ICAO marking agents, and other explosives of interest.

The challenge in trace detection is not detection; it is the collection of the sample. The molecules of explosives clinging to the clothing of the bomb carrier need to be activated, swept away, and collected. For successful trace detection, the explosive sample must be collected from a surface or air stream, separated from all the background, detected, and identified. Current trace detection technology requires intimate contact with surfaces for residues of low vapor pressure military explosives. Some systems employ fast (typically 5-10 s) gas chromatography to separate the explosive molecules collected from all the other chemicals that may interfere with the detection. Trace explosive detection systems have been operationally evaluated in airports. Trace detectors are routinely used to examine electronic items for concealed explosives, which is a difficult task for $\mathrm{x}$ ray systems with human operators. The false alarm rate is less than $0.2 \%$, with a majority of the false alarms attributable to the legitimate presence of explosive residues. The FAA is in the process of purchasing over 400 trace detectors, costing between $\$ 45,000$ and $\$ 160,000$ each, in FY-97 for deployment in United States airports. Trace detection technology continues to advance for the screening of people as well, although how to do it quickly without upsetting the billion plus people that fly every year is a challenge for the FAA. 
Trace detection systems have been used in airports in Canada, Germany, other locations and to protect selected federal installations. The same detection technology is being incorporated into walk-through portals that can be used to screen people for concealed explosives and into a portable car mount to operate at a vehicle checkpoint of opportunity. Some systems have portable sample collecting systems to clear suspect packages. The ability of trace detectors to detect volatile explosives, low volatility explosives, and ICAO markers make them a powerful detection tool with two complementary mechanisms for the detection of high threat plastic explosives: the volatile marker or the nonvolatile explosive. All of these attributes can carry over directly to military applications.

Electron capture. Early commercial explosive vapor detection systems employed electron capture detectors (ECD) to detect volatile explosives, specifically, nitroglycerin and ethylene glycol dinitrate, which are present at high vapor concentrations around many 1970's dynamites. These systems employed preconcentration, semipermeable membranes and/or gas chromatography to separate the explosive molecules from the electronegative components of air. Explosives are very electronegative, that is, they easily capture electrons, which ECD exploits as a sensitive detection mechanism. However, compounds other than explosives are electronegative. Current commercial trace detectors have moved away from ECD for explosive detection because of its lack of specificity and the resulting false alarm problem.

Chemiluminesence is a nitrogen specific detector. Explosive molecules containing nitrogen are separated using gas chromatography from the rest of the materials collected from the air. Once separated, they are pyrolysed to give NO that is reacted with ozone to give excited NO2 that emits infrared radiation. The approach is very sensitive. Specificity is gained by chromatography.

Ion Mobility Spectroscopy separates explosive molecules from the air background by gas chromatography and time of flight. The electronegative explosive molecule is introduced into the system and ionized by attaching an electron or a small charged molecule. Most molecules in air are not as electronegative as explosives; therefore, they are not ionized. The charged explosive molecule is carried into an electrostatic field and accelerated. Its time of flight to move through a counter-current drift gas and reach the collecting electrode is measured and is characteristic of its mobility. Detection is made by averaging over hundreds of these fast events. There are several commercial vendors of trace explosive detectors employing IMS.

Mass Spectroscopy is theoretically the ideal instrument to use as an explosive detector, as it should provide instantaneous identification of molecules based on their fragmentation pattern and mass. Although this approach has worked in the laboratory, the cost, complexity and demands of a vacuum system have kept this technology out of the commercial market.

Antibodies are the protective cells formed in the body in response to the introduction of foreign materials. They are also formed in response to the introduction of explosives and the chemicals used in their production. Such antibodies can be detected with the research tools of modern biochemistry. However, those tools are not yet fully developed and would probably require measurement in the bloodstream to provide the biological or genetic materials required for testing. Such tests would be more invasive than others suggested above, and their evaluation could be much more time consuming.

Layered, synergistic approaches are appropriate, as none of the technologies discussed above are ideal by themselves. As noted above, a chemical detection system that detected both explosive and carrier could be much more effective than one that detected only one or the other. And a system that used a combination of $\mathrm{x}$-ray tomography for search and trace detection for confirmation could largely eliminate the weaknesses of both.

What are the limitations?

Explosives can be detected by exploiting the bulk properties or the detailed chemistry of suspect objects. The former is generally faster but less discriminating; the latter is generally more specific but more time consuming and expensive. The optimum combination of techniques has not been found in the FAA program, compared to which the current DoD program is rudimentary. The paragraphs below discuss the limitations of current technologies, and the additional limitations introduced by $\mathrm{DoD}$ applications.

The military search and detection capability is based on inspection and canine olfaction. 
Inspection is susceptible to concealment and deception, which produces low detection rates and low throughput. Dogs are the classic trace detection system. They can detect the characteristic scent of explosives and other ingredients of explosives. They are used successfully for land mine clearance and explosive detection, but tire and lose interest in routine operations. However, they are an ideal tool for perimeter and area searches, if breaches are detected.

$x$-ray detection is a well developed capability, which is adequate for simple shadowgraphs of readily recognizable objects. It works well for high contrast targets such as handguns but not low-contrast targets like explosives. Dual energy systems provide more contrast, but not enough for automatic recognition. An impediment to the wider use of automatic $\mathrm{x}$-ray systems is their cost, which currently about $\$ 350,000$.

$x$-ray tomography has been certified as an automated Explosives Detection System. It has demonstrated the ability to detect threat quantities of a broad range of commercial and military explosives and is deployed at airports in the US and abroad. Over 50 units are being purchased at about $\$ 900,000$ each to screen checked airline baggage. It is unlikely that such units could achieve the cost, size, and mobility goals of military portal systems, let alone search systems.

Thermal neutrons. react with nitrogen atoms in commercial and military explosives to give a hard gamma ray, which stands out from the background, allowing an estimate of nitrogen content. However, innocent objects with high nitrogen densities cause false alarms. In operational tests, thermal neutron systems' performance and operational availability were good, but they were not accepted because of size, cost and limited ability to address explosives smaller than about $1 \mathrm{~kg}$.

For military systems explosive charges are likely to be much larger and truck transport is a favored option; thus, the limitation to $>1 \mathrm{~kg}$ is not as severe. Thermal neutrons could be effective in military applications, if the neutron sources and gamma ray detectors are small and cheap, which has not been established by civil programs.

Fast neutrons allow in situ elemental analysis, in which explosives can be recognized by their characteristic elemental ratios of oxygen, carbon and nitrogen. Sealed tube generators simplify the construction of the source and the timing of the pulse at the expense of flux. Pulsed fast neutrons complicates the source. Transmission shadowgraphs require measurement of the transmitted spectrum. All three approaches are in the experimental stage. The pulsed fast neutron approach is the most mature. It has been used in the laboratory, and an operational prototype is under construction. The main issues with fast neutrons are their immaturity and sensitivity. As with thermal neutron systems, a limitation to $>1 \mathrm{~kg}$ of explosive is not debilitating.

Quadrupole Resonance has good detection rates and low false alarm rates because of its elemental specificity. It requires neither massive radiation shielding nor sophisticated image analysis software. However, it must interrogate the sample with the correct frequency and pulse train shape for each explosive of interest. Thus far, it is available commercially in small systems for RDX and PETN. The cost and mobility of more flexible systems have not been established.

Trace detection. Current technology is capable of simultaneously detecting and identifying less than one nanogram of RDX or PETN and other explosives. The challenge is sample collection. Current technology requires intimate contact with surfaces for residues of low vapor pressure military explosives. Trace detection has been operationally evaluated with false alarm rates $<0.2 \%$. The FAA is purchasing over 400 trace detectors for $\$ 45,000-160,000$ each for deployment in airports. The same detection technology is being incorporated into walk-through portals to screen people for concealed explosives. Their ability to detect both volatile marker and nonvolatile explosives increases detection and selectivity. While costs are slightly higher than desired for military applications, the more serious barriers would appear to be the low throughput ( $30 \mathrm{~s} /$ person and $60 \mathrm{~s} / \mathrm{vehicle}$ ), size, complexity, and lack of mobility.

Electron capture. is effective in detecting volatile explosives such as nitroglycerin. However, compounds other than explosives are electronegative, which increases the false alarm rate, and modern explosives have much less volatility, so current commercial trace detectors have moved away from ECD because of its lack of specificity and false alarm rate. Thus, it would be of only limited use for military applications.

Chemiluminesence is nitrogen specific and very sensitive in combination with chromatography. However, the resulting analytical laboratory is complex and expensive. 
Ion Mobility Spectroscopy has greater specificity than electron capture alone, but still requires direct sampling and involves a more complex instrument.

Mass Spectroscopy works in the laboratory, but the cost, complexity and demands of a vacuum system have kept this technology out of the commercial market. These factors would be even more of a barrier to military applications.

Antibodies form in response to the introduction of explosives and the chemicals used in their production. They can be detected with the research tools of modern biochemistry. However, those tools are not completely developed, and require measurement in the bloodstream to provide the biological or genetic materials required for testing. Such tests would be more invasive than others suggested above, and their evaluation could be much more time consuming.

Layered, synergistic approaches are desirable. None of the concepts discussed above are ideal by themselves. As noted above, a chemical detection system that detected both explosive and carrier could be much more effective than one that detected only one or the other. And a system that used a combination of $\mathrm{x}$-ray tomography for search and trace detection for confirmation could largely eliminate the weaknesses of both. However, it is difficult to build a synergistic combination of the simplest systems. For example, one energy, two energy, and automated $x$-ray systems with a detection probability of $p \sim 0.7$ and false alarm probability $f \sim 0.5$ might cost $\$ 50, \$ 150$, and $\$ 350 \mathrm{~K}$, respectively. Thus, it would be attractive to use a one energy sensor as a screening device for an automated $x$-ray systems, but the resulting combination would cost $\sim \$ 400 \mathrm{~K}$, have a probability of detection $\sim 1-(1-.7)^{\wedge} 2 \sim 0.9$, but a false alarm rate of $\sim 1-(1-.5)^{\wedge} 2 \sim 0.75$, which is so high that it would essentially be necessary to re-inspect every parcel, person, or vehicle. For the better sensors, such as $\mathrm{x}$-ray tomography, neutrons, quadrupole resonance, and trace detection, it might be possible to create useful layered systems, but they appear to be large, complex, expensive, slow, and immobile. Thus, the more capable systems might be deployed better by themselves, as a simple screening sensor would not appear to add value commensurate with its cost in military applications.

What is the new approach?

The new approach involves steps ranging from the simple and familiar to the more complex and developmental. A number involve the technologies above, but used in a manner that avoids their limitations in civil applications.

Measuring mass. The first is to weigh vehicles as they come through a portal. Any significant amount of explosive, however hidden to the eye and other sensors, should manifest itself as an anomalous vehicle weight, which could serve as a high-confidence indicator for further screening. Weighing could either be done with a typical weight and balance scale, as used for commercial trucks on U.S. highways, or with a series of speed bumps-comparing the trucks actual response to its expected response would give an indirect but confident estimate of its mass while permitting greater throughput.

$x$-rays and neutrons for large vehicles. A second measure is imaging large vehicles with $\mathrm{x}$ rays and thermal or fast neutrons. These sensors are less favored by civil investigators because they are limited in sensitivity to $\sim 1 \mathrm{~kg}$ of explosive. While that might be a lethal amount for an aircraft, much larger explosives are needed to destroy military facilities, so an amount this small could be a very useful threshold for a military sensor. With such a range between the likely $1,000 \mathrm{~kg}$ payload and this $\sim 1 \mathrm{~kg}$ threshold, the resulting $\sim 1,000 / 1$ signal to noise ratio should support confident detection with one or more of the simpler sensors.

Screening. Another measure is to pre-screen and/or profile persons approaching portals. This step could involve a number of steps from manual or automated template matching to the establishment of computer files on individuals who frequently enter or attempt to enter facilities.

Trace detection should be practical on military facilities. Those who present themselves at portals are requesting entry. To support that application, they are expected to surrender certain articles such as identity cards, briefcases, and the like and subject themselves to simple searches. They afford the opportunity for direct contact, which is the most difficult step in trace detection, as discussed above. Given that the surrendered items can be swabbed in a few tens of seconds to extract samples, if the simple chemical processing required for trace detection can be effected in the few minutes of current automated systems, it should be possible to fully screen the individual for 
contact with all explosive materials of interest within the $\sim$ few minute cycle for clearance onto military facilities in foreign countries.

Smart nose. Trace detection could be simplified, and its throughput greatly increased, by the development of "smart nose" technologies, i.e., enzyme mimics and the class of semiconductor array sensors that can do molecular recognition with accuracies approaching those of the dog's nose-without tiring and without loss of attention or sensitivity. While this technology could take 5-10 years to develop, it would represent a fundamental step towards the advanced trace detection sensors for confident detection in affordable packages for proliferated or mobile deployment.

Chemistry on a chip and associated MEMS technologies offer the promise of compact, rugged, and affordable analytical laboratories that could be used in mobile micro-platforms for remote detection of chemical compounds or in distributed arrays for the gathering of intelligence. Although this is probably also on the 5-10 year time scale, it would provide flexible, throw away sensors capable of detecting new threats as well as established one. Semiconductor implementation should make mass production of such sensors feasible.

Canine olfaction. The implementation of the above measures would put the burden of routine search and detection on automated sensors, which would release dogs for the tasks they are best at: area search, quick scans of new areas, detection of distributed supplies of C4, Semtex, and other low volatility explosives, and novel missions for which dogs do not get bored.

Why will it be successful?

Measuring mass. Mass is the fundamental quantity that is most difficult to conceal. As always, it is the best discriminant. A scale is the simplest and fastest way to determine it. Thus, it can serve as a high-confidence indicator for further screening. Knowing the mass in conjunction with a X-ray or neutron image leaves little freedom for an intruder to hide explosives.

$x$-rays and neutrons for large vehicles. Large explosives are needed to destroy military facilities, so a threshold of $\sim 1 \mathrm{~kg}$ is very useful threshold for military applications. Its signal to noise ratio should support confident detection with one or more of the simpler sensors.

Screening persons approaching portals has been shown to be effective. It could involve steps from manual or automated template matching to the establishment of computer files on individuals who frequently enter or attempt to enter facilities. The computational burden for comparison and data exchange should not be burdensome.

Trace detection should be practical on military facilities as those who request entry must surrender articles that afford the opportunity for direct collection, which is the most difficult step in trace detection. It appears technically feasible to process them in a few minutes, making it possible to screen individuals for contact with explosives within the clearance cycle for admission.

Smart nose. Enzyme mimics and semiconductor array sensors for molecular recognition are within 5-10 years of development. They represent a fundamental step towards trace detection sensors in affordable packages for proliferated or mobile deployment.

Chemistry on a chip offers compact, rugged, affordable analytical laboratories on mobile micro-platforms for remote detection or distributed arrays for within 5-10 years of development.

Canine olfaction for facility sweeps. The implementation of the above measures would put the burden of routine search and detection on automated sensors, which would release canine olfaction for the tasks it is best at: novel missions for which dogs have long proven their value.

If successful, what is the payoff? Successful pursuit of the technologies and concepts discussed above would make it possible to secure the boundaries of military facilities against attempts to infiltrate high explosives and permit the rapid sweep of its perimeter and interior should such infiltration occur.

Measuring mass would provide a high quality discriminant and indicator for further screening, which would leave an intruder little freedom to hide explosives.

$x$-rays and neutrons would provide high signal to noise portal search and detection for large vehicles as well as a low false alarm screen for further inspection.

Screening persons approaching portals with template matching to computer files is a proven technique, which is now computationally feasible for real time denial or reaction.

Trace detection on military facilities is simplified by the requirement to surrender articles that permit direct collection, which is the most difficult step. Processing should be possible within 
the normal clearance cycle for admission at reasonable throughput.

Smart nose technologies represent a fundamental step towards trace detection in affordable packages for proliferated or mobile deployment.

Chemistry on a chip offers compact, rugged, and affordable analytical laboratories on mobile micro-platforms for remote detection or distributed intelligence arrays.

Canine olfaction for facility sweeps. The implementation of the above measures would permit the proper reallocation of dogs for the novel missions at which they have long proven their value. 